\title{
Infrared-induced conformational interconversion in carboxylic acids isolated in low-temperature rare-gas matrices
}

\author{
Ermelinda M.S. Maçôas ${ }^{\text {a,b,*, Leonid Khriachtchev }}{ }^{\mathrm{a}}$, Mika Pettersson ${ }^{\mathrm{a}}$, \\ Jan Lundell $^{\mathrm{a}}$, Rui Fausto ${ }^{\mathrm{b}}$, Markku Räsänen ${ }^{\mathrm{a}}$ \\ ${ }^{a}$ Department of Chemistry, University of Helsinki, P.O. Box 55 (A.I. Virtasen aukio 1), Helsinki FIN-00014, Finland \\ ${ }^{\mathrm{b}}$ Department of Chemistry (CQC),University of Coimbra, Coimbra P-3004-535, Portugal
}

Received 17 March 2003; received in revised form 10 July 2003; accepted 11 July 2003

\begin{abstract}
An overview of our recent studies dealing with infrared-induced conformational interconversion of carboxylic acids isolated in rare-gas matrices is presented. Extensive rotational photoisomerization studies have been performed on formic acid, which is the simplest organic acid enabling this kind of processes. Formic acid has two conformers and interconversion between them can be induced by vibrational excitation. As such, it is an ideal model system to study the conformational dynamics of the carboxylic group. Formic acid molecules were found to be isolated in different local environments within the rare-gas matrices, as shown by the site splitting of the vibrational bands. Narrowband tunable infrared (IR) radiation was used to induce site-selective isomerization processes. The induced changes in the IR absorption spectra allowed for a detailed analysis of the vibrational properties of both conformers of formic acid isolated in solid argon. In particular, derived from the intermode coupling constants the local environment was shown to affects the intramolecular potential energy surface. Tunneling is involved in the rotamerization of formic acid, with the tunneling rate being affected by the local environment. Additionally, formic acid exhibits isomer-selective photodissociation where narrowband IR excitation can control the conformer-dependent photodissociation channels. Tunable IR radiation was also used to promote rotamerization in a series of matrix-isolated dicarboxylic acids (ethanedioic, propanedioic, and 2-butenedioic acids) by exciting the first overtone of the $\mathrm{O}-\mathrm{H}$ stretching mode or a suitable combination mode at similar energies. Efficient isomerization involving rotation around the $\mathrm{C}-\mathrm{O}$ bond was observed in most cases whereas the internal rotation around the $\mathrm{C}-\mathrm{C}$ bond was found to be constrained for ethanedioic and (Z)-2-butenedioic acids.
\end{abstract}

(C) 2003 Elsevier B.V. All rights reserved.

Keywords: Vibrational excitation; Energy relaxation; Conformational interconversion; Matrix-isolation

\section{Introduction}

The low-temperature matrix-isolation technique combined with various spectroscopic methods (in particular, IR spectroscopy) is a powerful approach to the characterization of conformational properties of molecules. In a matrix-isolation experiment the low temperature of the sample suppresses thermal reactions with significant activation energy, hence enabling stabilization of higher energy conformers. Very often the conformational distribution of the gaseous sample (prior to deposition) is trapped in the matrix upon freezing the sample [1]. If the energy barriers are sufficiently low, the annealing of the matrix may induce conformational conversion of the higher energy species into the lower energy ones.

\footnotetext{
* Corresponding author.

E-mail address: emacoas@qui.uc.pt (E.M.S. Maçôas).
}

However, investigation of thermally induced isomerization reactions is possible only within a very limited range of energy barriers due to matrix-temperature limitations [1]. In contrast, photoinduced rotational isomerization is only limited by the photon energy.

A considerable number of studies have been dedicated to IR-induced rotamerization in low-temperature matrices [1-9]. Most of the earlier studies used broadband excitation, which can only be selective to some extent. However, a few examples are known in which narrowband irradiation was involved [10-16]. The limited number of systematic studies performed using narrowband selective vibrational excitation might be partially attributed to the lack of available tunable IR sources. The use of narrowband IR irradiation allows to excite selectively a specific vibrational mode of a desired conformer. This approach can be used to probe mode-selectivity of vibrational energy relaxation pathways. 


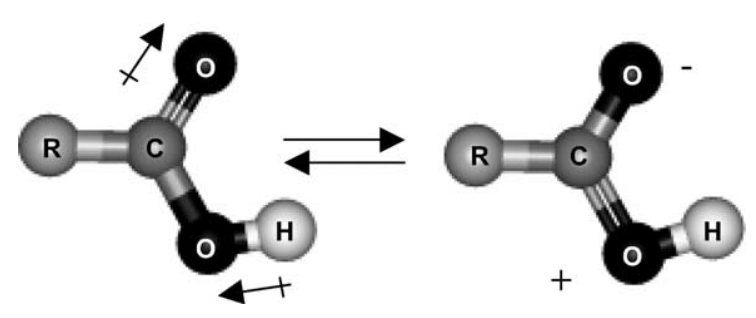

Fig. 1. The $\mathrm{O}=\mathrm{C}-\mathrm{O}-\mathrm{H}$ dihedral angle of $0^{\circ}$ is stabilized by a higher efficiency of the mesomerism within the carboxylic group $[\mathrm{O}=\mathrm{C}-\mathrm{O}-\mathrm{H} \leftrightarrow$ $\left.\mathrm{O}^{-}-\mathrm{C}=\mathrm{O}^{+}-\mathrm{H}\right]$ and by an attractive interaction between the $\mathrm{C}=\mathrm{O}$ and $\mathrm{O}-\mathrm{H}$ dipoles when compared with the $180^{\circ}$ dihedral angle. Arrows indicate the direction of the dipoles.

Splitting of IR absorption bands of matrix-isolated species is a well-known phenomenon usually called a matrix-site effect. This splitting is caused by different local environments of the trapped species and potentially provides a way to study local matrix morphology and its effects on the molecular properties. Narrowband radiation can be used to pump selectively molecules [e.g. $\mathrm{HONO}$ and $(\mathrm{FCO})_{2}$ ] isolated in various matrix sites $[15,16]$.

In the present work, we focus on the photoinduced rotamerization of matrix-isolated carboxylic acids. An isolated carboxylic group adopts preferentially a planar geometry. When compared with the $180^{\circ}$ arrangement of the $\mathrm{O}=\mathrm{C}-\mathrm{O}-\mathrm{H}$ dihedral angle, the $0^{\circ}$ arrangement is stabilized by a higher efficiency of the mesomerism within the carboxylic group $\left[\mathrm{O}=\mathrm{C}-\mathrm{O}-\mathrm{H} \leftrightarrow \mathrm{O}^{-}-\mathrm{C}=\mathrm{O}^{+}-\mathrm{H}\right]$, an attractive interaction between the $\mathrm{C}=\mathrm{O}$ and $\mathrm{O}-\mathrm{H}$ bond-dipoles, and the absence of oxygen lone electron pair repulsion [17] (see Fig. 1). The interconversion between the two stable planar forms of the carboxylic group occurs via rotation of the $\mathrm{O}-\mathrm{H}$ bond around the $\mathrm{C}-\mathrm{O}$ axis. Since the reaction coordinate is mainly described by the motion of the hydrogen atom, tunneling can be important for the conversion mechanism. In fact, tunneling from the higher energy cis conformer of formic acid to the lower energy trans form was observed when the cis conformer was prepared in an Ar matrix by IR pumping [18]. The recently published studies on formic acid have further emphasized the importance of tunneling in the isomerization reactions $[19,20]$. Additionally, isomerization studies performed on hydroquinone and on a halogenated derivative of dihydroxy-benzoquinone isolated in argon matrices $[21,22]$ seem to indicate that the participation of tunneling in isomerization processes involving mainly motion of hydrogen atoms is quite general. Solid-state medium is expected to influence the tunneling rate [23-25], which depends also on the temperature due to the participation of the lattice phonons in a dissipative tunneling mechanism [26-28].

Isomer-selective photochemistry has motivated many works on IR-induced rotamerization. In addition to its fundamental value, isomer-selective photochemistry is a potential way to optically control chemical reactions. This is possible if the reaction occurs before the excitation energy is transferred to the coordinate that leads to rotamerization.
For instance, geometrical considerations were suggested to explain the high $\mathrm{CO} / \mathrm{CO}_{2}$ yield ratio for the photodissociation of trans-formic acid in the gas phase [29], indicating that the dissociation is faster than conformational randomization. Experimental evidence of isomer-dependent photodissociation channels has also been recently reported for $1-\mathrm{C}_{3} \mathrm{H}_{7} \mathrm{I}^{+}$in the gas phase, where the products resulting from dissociation of the gauche and anti isomers correspond to different $\mathrm{C}_{3} \mathrm{H}_{7}{ }^{+}$isomeric forms [30,31].

The aim of this paper is to describe our studies on conformational isomerization reactions induced on carboxylic acids by selective vibrational excitation. Formic acid was used as model system for investigation of the conformational dynamics of the carboxylic group. First, we will discuss the use of vibrational spectroscopy coupled to narrowband tunable excitation for conformational identification. This photoreactive spectroscopic method is useful to study the vibrational properties of matrix-isolated formic acid conformers, in particular, the local environmental effects on mode coupling [32]. Next, the trans $\rightarrow$ cis isomerization induced by mode selective excitation in formic acid is addressed [20]. Our discussion is focused here on the high quantum yields observed for excitation energies below the torsional energy barrier. This is followed by the analysis of the influence of the medium on the tunneling mechanism that leads to the dark cis $\rightarrow$ trans formic acid conversion [19]. Additionally, isomer-selective UV-photodissociation of formic acid is described [33]. Finally, the conclusions drawn from photoinduced rotamerization studies on dicarboxylic acids [34-36] are presented.

\section{Site-selective vibrational spectroscopy}

Formic acid monomer exhibits two stable planar structures, the cis and the trans conformers shown in Fig. 2. Trans-formic acid has the hydrogen atoms on opposite sides of the $\mathrm{C}-\mathrm{O}$ bond $\left(\mathrm{H}-\mathrm{C}-\mathrm{O}-\mathrm{H}\right.$ dihedral angle of $\left.180^{\circ}\right)$, while in the cis conformer the hydrogen atoms are located on the same side of the $\mathrm{C}-\mathrm{O}$ bond. The trans form is the most stable and hence predominant in the gas phase and in the asdeposited matrix samples. Excitation of the first overtone of the $\mathrm{OH}$ stretching mode $(2 \mathrm{vOH})$ of trans-formic acid isolated in $\mathrm{Ar}$ was earlier shown to promote trans $\rightarrow$ cis rotamerization [18]. On the other hand, the cis $\rightarrow$ trans conversion was shown to take place in the dark at low temperatures via a tunneling mechanism [18]. In the absence of optical pumping, this back-reaction limits the detectability of $c i s$-formic acid after its preparation, even at the low working temperatures $(4-60 \mathrm{~K})$ used in rare-gas matrix studies.

Recently, other vibrational modes were excited to induce the trans $\rightarrow$ cis isomerization [20]. Formic acid molecules are isolated in different local environments of the rare-gas matrices, as shown by the site splitting of the IR absorption 
TS

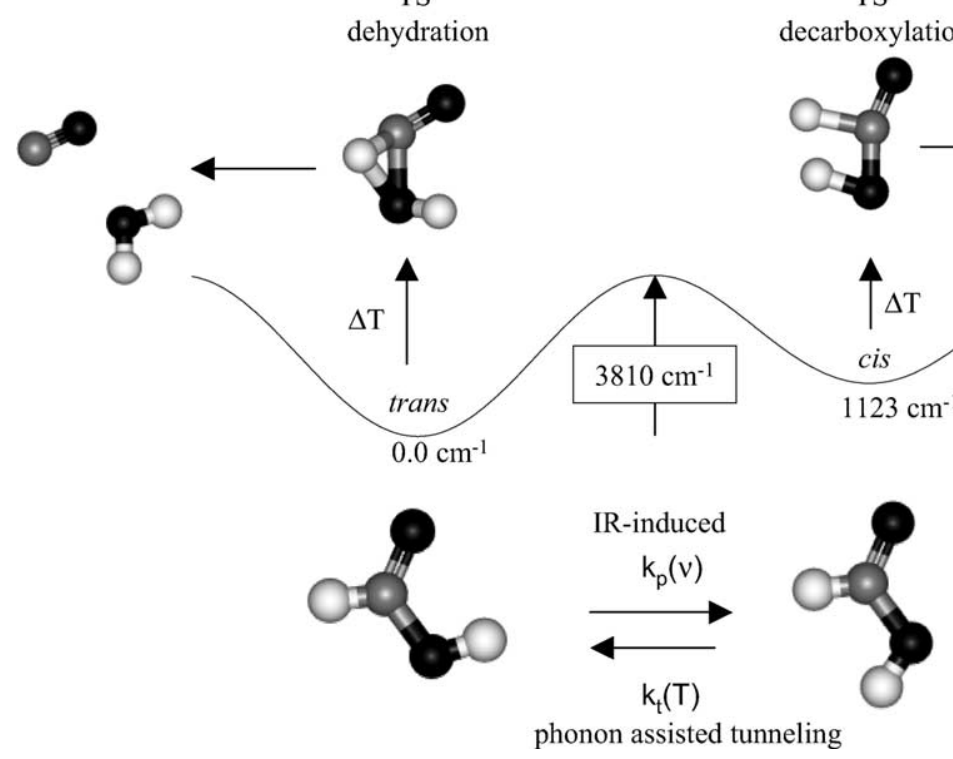

Fig. 2. Formic acid conformers. Energy difference and energy barrier estimated for the molecule isolated in solid Ar are shown as estimated at the CCSD(T)/ aug-cc-pVTZ//MP2/aug-cc-pVTZ level for the molecule in vacuum and corrected by the PCM solvation model [19]. The transition state geometry for thermal $(\Delta T)$ dehydration and decarboxylation reaction are shown in connection with the trans and cis conformer, respectively. The predicted energy barrier for these reactions starting form the corresponding conformers is $280 \mathrm{~kJ} \mathrm{~mol}^{-1}$ [38].

bands [32]. Pulsed IR radiation with a spectral linewidth of $\approx 0.1 \mathrm{~cm}^{-1}$, provided by an optical parametric oscillator (Continuum), was used to promote the site-selective trans $\rightarrow$ cis rotamerization $[19,32]$. In order to maintain a sufficiently high concentration of the less stable cis conformer in the matrix, the IR absorption spectra were recorded under IR pumping of the trans form [32]. Without pumping, the concentration of the cis conformer produced by IR-irradiation decreases by a factor of three due to the cis $\rightarrow$ trans tunneling during the time needed to record a spectrum at $0.25 \mathrm{~cm}^{-1}$ resolution with a reasonable signal/noise ratio. Such a spectral resolution is needed to fully resolve the sitestructure of the observed bands [32].

By comparing the IR absorption spectra recorded under pumping and the spectra of the as-deposited (non-irradiated) sample, we can identify bands belonging to each conformer isolated in a particular site, as illustrated in Fig. 3. As seen in this figure, the $\mathrm{OH}$ and $\mathrm{C}=\mathrm{O}$ stretching vibrations of cis formic acid are shifted to higher wavenumbers from their trans values and the site splitting of the bands is resolved.

Detailed vibrational analysis of the near-IR region allowed for the identification of various overtone and combination bands [32]. Based on this assignment we have calculated site-selective anharmonicities and coupling constants for several modes of both conformers. The determined values for the trans conformer are presented in Table 1. By comparing the coupling constants obtained for this conformer in solid argon with the gas phase data [37], it was concluded that the cage potential affects the guest intermode couplings. The changes in the coupling constants are in many cases of similar magnitude as the constants themselves. Moreover, the anharmonicity changes between molecules isolated in different sites (see, for example, the constants of $v \mathrm{CH}+v \mathrm{C}=\mathrm{O}$ or $\mathrm{vCH}+\gamma \mathrm{CH}$ modes in Table 1). Thus, one can expect the local environment to affect the vibrational energy relaxation pathways in condensed phase, which depend on mode couplings [38].

Table 1

Observed anharmonicities and coupling constants ${ }^{\mathrm{a}}$ for trans-formic acid

\begin{tabular}{|c|c|c|c|}
\hline \multicolumn{4}{|l|}{ trans-HCOOH } \\
\hline \multirow[t]{2}{*}{ Combinations } & Site 1 & Site 2 & Gas phase ${ }^{b}$ \\
\hline & \multicolumn{3}{|c|}{$\overline{\text { Coupling constants }\left(x_{i j}, \mathrm{~cm}^{-1}\right)}$} \\
\hline$\overline{v \mathrm{OH}+v \mathrm{C}=\mathrm{O}}$ & -0.7 & -0.5 & -1.2 \\
\hline$\nu \mathrm{OH}+\gamma \mathrm{CH}$ & -3.9 & -5.4 & -3.6 \\
\hline$v \mathrm{OH}+\mathrm{COH}-\mathrm{CO}$ def. & -2.5 & -2.3 & -1.8 \\
\hline$v \mathrm{OH}+\omega \mathrm{CH}$ & & & -1.0 \\
\hline$\nu \mathrm{OH}+\tau \mathrm{COH}$ & (0) & $(-0.7)$ & $(-2.2)$ \\
\hline$\nu \mathrm{OH}+\delta \mathrm{OCO}$ & $(-15.5)$ & & $(-4.7)$ \\
\hline$v \mathrm{CH}+v \mathrm{C}=\mathrm{O}$ & -18.0 & -14.3 & -5.4 \\
\hline$v \mathrm{CH}+\gamma \mathrm{CH}$ & -23.5 & -17.1 & -22.1 \\
\hline$v \mathrm{CH}+\mathrm{COH}-\mathrm{CO}$ def. & -0.3 & -1.0 & -1.8 \\
\hline$v \mathrm{CH}+\omega \mathrm{CH}$ & & & -5.7 \\
\hline$v \mathrm{C}=\mathrm{O}+\mathrm{COH}-\mathrm{CO}$ def. & & -4.8 & -2.6 \\
\hline$v \mathrm{C}=\mathrm{O}+\tau \mathrm{COH}$ & $(-6.4)$ & $(-5.5)$ & \\
\hline Overtones & \multicolumn{3}{|c|}{ Anharmonicity constants $\left(x_{i i}, \mathrm{~cm}^{-1}\right)$} \\
\hline $2 v \mathrm{OH}$ & -83.1 & -83.1 & -91.2 \\
\hline $2 v \mathrm{CH}$ & -53.1 & & -52.4 \\
\hline $2 v \mathrm{C}=\mathrm{O}$ & -9.4 & -9.4 & -9.3 \\
\hline $2 \mathrm{COH}-\mathrm{CO}$ def. & -5.9 & -5.7 & -6.6 \\
\hline
\end{tabular}

${ }^{\mathrm{a}} x_{i j}=v_{i+j}-v_{i}-v_{j} ; x_{i i}=\left(2 v_{i}-2 x v_{i}\right) / 2$.

${ }^{\mathrm{b}}$ Gas phase data was taken from [36]. Values in parentheses were calculated based on a tentative assignment. Values centred between site 1 and site 2 columns are average estimates for the two sites. 

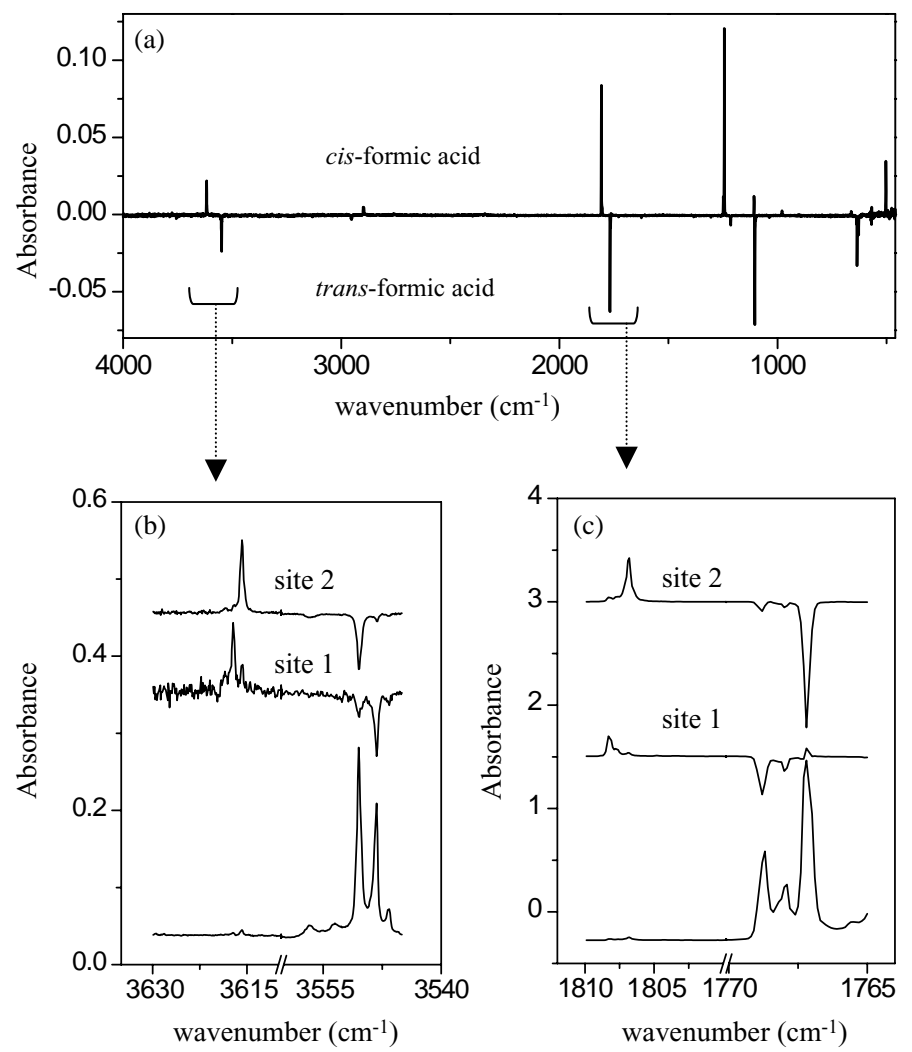

Fig. 3. IR absorption spectra of formic acid isolated in an Ar matrix. (a) Difference spectrum showing the result of pumping the overtone of the OH stretching of trans-formic acid, i.e. the increase of bands of the cis conformer and the decrease of the bands of the trans conformer. In (b) and (c) the site-selectivity of the IR-pumping is shown for the $\mathrm{OH}$ and $\mathrm{C}=\mathrm{O}$ stretching regions. The lower spectrum show the bands of trans-formic acid present in the as-deposited sample. The difference spectra [the two upper traces of (b) and (c)] show the result of the excitation of different sites of formic acid, where the bands of the cis conformer isolated in the pumped site increase while the ones of the trans conformer decrease. (a)-(c) were obtained in experiments performed with different samples.

\section{Rotamerization quantum yield as a function of the excitation energy}

The quantum yields for the trans $\rightarrow$ cis reaction in formic acid were measured upon excitation of several vibrational modes of the trans conformer in the $2900-4700 \mathrm{~cm}^{-1}$ spectral range [20]. In the calculations, the cis $\rightarrow$ trans back reaction was taken into account (see Fig. 2). The quantum yield for the rotamerization process is determined from the trans $\rightarrow$ cis pumping efficiency, the absorption cross-section of the excited mode of the trans conformer and the intensity of the excitation radiation. The last two values are measured experimentally while the pumping efficiency is calculated on the basis of the tunneling rate coefficient and the photoequilibrium concentration ratio of the two conformers under mode-selective pumping.

The quantum yield as a function of the excitation energy is shown in Fig. 4. Interestingly, all excited modes led to an efficient energy relaxation into the reaction coordinate (torsion around the $\mathrm{C}-\mathrm{O}$ bond), resulting in rotational isomerization. The estimated energy barrier for the trans $\rightarrow$ cis rotamerization in formic acid isolated in solid $\mathrm{Ar}$ is $3810 \mathrm{~cm}^{-1}$ [20]. For excitation energies above this barrier, the average isomerization quantum yield is $\approx 20 \%$, without remarkable changes associated with the nature of the excited modes.

In addition to the importance of the tunneling mechanism in the cis $\rightarrow$ trans process, its role on the trans $\rightarrow$ cis photoinduced rotamerization is evident from experiments with pumping below the energy barrier. Despite that

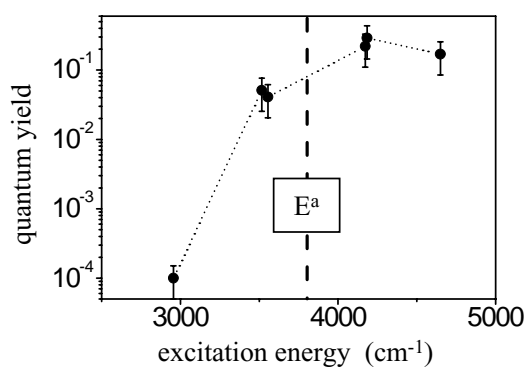

Fig. 4. Quantum yield for the trans $\rightarrow$ cis? Isomerization process of formic acid isolated in solid Ar as a function of the excitation energy. The points correspond to the following vibrational modes: $4651 \mathrm{~cm}^{-1}: \mathrm{vOH}+$ COH-CO def.; $4184 \mathrm{~cm}^{-1}: \mathrm{vOH}+\tau \mathrm{COH} ; 4174 \mathrm{~cm}^{-1}: \mathrm{vCH}+\mathrm{CO}-\mathrm{COH}$ def.; $3552 \mathrm{~cm}^{-1}$ : vOH; $3516 \mathrm{~cm}^{-1}: 2 v \mathrm{C}=\mathrm{O} ; 2955 \mathrm{~cm}^{-1}: \mathrm{vCH}$. The ab initio estimation for the energy barrier of the solvated molecule is indicated as a vertical dashed line at $E^{a} \approx 3810 \mathrm{~cm}^{-1}$ [19]. The line connecting the points is for guiding the eyes. 
excitation of the $\mathrm{O}-\mathrm{H}$ stretching mode $\left(\mathrm{vOH}\right.$ at $\left.3549 \mathrm{~cm}^{-1}\right)$ or the first overtone of the $\mathrm{C}=\mathrm{O}$ stretching mode $(2 \mathrm{vC}=\mathrm{O}$ at $3516 \mathrm{~cm}^{-1}$ ) is energetically below the torsional barrier [20], the quantum yield decreases only by a factor of 4 when compared with the values for the higher energy modes. This high quantum yield was suggested to be due to the strong delocalization of the torsional wavefunction between the two potential wells at these energies [20]. The extent of delocalization decreases rapidly with the distance from the top of the energy barrier. In agreement with this, the measured rotamerization quantum yield upon excitation of the $\mathrm{C}-\mathrm{H}$ stretching mode $\left(\mathrm{vCH}\right.$ at $\left.2955 \mathrm{~cm}^{-1}\right)$ is about three orders of magnitude smaller than those at energies above the torsional barrier but still very reliably measure.

\section{Environment and temperature effects on phonon-assisted tunneling}

Even in a low-temperature matrix the cis conformer of formic acid decays spontaneously to the most stable trans conformer by phonon-assisted tunneling $[18,19]$. In this section, the effect of the matrix environment on the rate of the cis $\rightarrow$ trans tunneling is described [19]. The temperature-dependent tunneling rates for formic acid were measured in solid $\mathrm{Ar}, \mathrm{Kr}$ and $\mathrm{Xe}$. This was done by generating $c i s$-formic acid via optical pumping and then following the decay kinetics by IR absorption spectroscopy [19]. The site selectivity of the process was also investigated. The Arrhenius plots for the cis $\rightarrow$ trans reaction rate of formic acid isolated in various rare-gas matrices are shown in Fig. 5.

The temperature effect on the cis $\rightarrow$ trans tunneling rate is essentially due to thermal excitation of the phonon modes that are coupled to the tunneling process. The lattice phonons compensate the energy mismatch between the torsional ground state of the cis conformer (that is the starting level

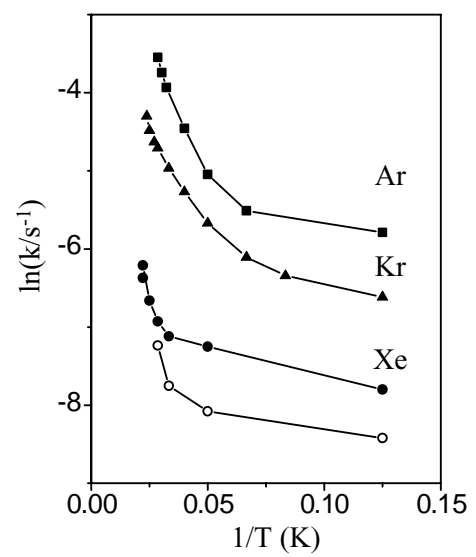

Fig. 5. Arrhenius plots for the cis $\rightarrow$ trans reaction rates of formic acid in rare-gas matrices $((\boldsymbol{\square}) \mathrm{Ar} ;(\boldsymbol{\Delta}) \mathrm{Kr}$ and $(\boldsymbol{O}),(\bigcirc) \mathrm{Xe})$. Solid and open symbols are used for the two predominant sites of the formic acid isolated in Xe. Molecules isolated in the different sites observed in $\mathrm{Ar}$ and $\mathrm{Kr}$ matrices exhibit no difference in the temperature dependence of the tunneling rate. for the tunneling) and the particular excited level of the trans conformer involved in the process. The specific temperature dependence of the rate constant originates from the type of phonon processes involved in the dissipative tunneling mechanism (e.g. emission, absorption or Raman processes $[26,27])$.

In order to understand the effect of the environment on the tunneling rate, it is important to know the position of the cis torsional ground state with respect to the energy level manifold of the trans conformer. This energy diagram determines the most probable states of the product participating in the tunneling. The energy gaps and the nature of the excited modes of the trans conformer involved in the process are factors affecting the cis $\rightarrow$ trans tunneling rate. The environment affects the potential energy surface of the guest molecules and, consequently, it can influence both factors. For example, the different solvation energy of the two formic acid conformers is expected to decrease their conformational energy difference in Xe when compared to $\mathrm{Ar}$ and $\mathrm{Kr}$ [19]. On the other hand, the cis $\rightarrow$ trans energy barrier was estimated to be higher in $\mathrm{Xe}$ than in Ar or $\mathrm{Kr}$ [19]. The different tunneling rates observed for the three studied hosts could be interpreted qualitatively on the basis of the estimated relative position of the cis torsional ground state with respect to the level manifold of the trans form [19].

Additionally, different local environments within the same host may have different solvation energies due to changes in the cavity size and geometry. Indeed, as shown in Fig. 5, the tunneling rate depends strongly on the host material and on the local morphology in solid Xe. Within our experimental error, the tunneling rates are site-independent when isolated in $\mathrm{Ar}$ and $\mathrm{Kr}$ matrices (see Fig. 5).

\section{Isomer-selective photodissociation}

The thermal unimolecular dissociation of formic acid can take place through decarboxylation and dehydration reactions [39] to produce, respectively, molecular hydrogen and carbon monoxide and carbon monoxide and water. As shown in Fig. 2, the transition states for these two reaction channels are geometrically connected either with cis-formic acid (decarboxylation) or trans-formic acid (dehydration) [39].

The theoretically predicted energy barriers for the dehydration and decarboxylation reactions on the $S_{o}$ potential energy surface of formic acid are similar $\left(\sim 23,500 \mathrm{~cm}^{-1}\right.$ [29]) whereas the energy barrier for the isomerization reaction is much smaller $\left(\leq 4000 \mathrm{~cm}^{-1}\right)$ [20]. In spite of that, a high $\mathrm{CO} / \mathrm{CO}_{2}$ photolysis branching ratio was observed after the $193 \mathrm{~nm}$ irradiation of trans-formic acid isolated in $\mathrm{Ar}$ and $\mathrm{Kr}\left(\mathrm{CO} / \mathrm{CO}_{2} \sim 5\right.$ and 4 in $\mathrm{Ar}$ and $\mathrm{Kr}$, respectively) [40]. An even higher branching ratio was observed for the gas-phase photodissociation after 193 and $248 \mathrm{~nm}$ irradiation [29]. The expected branching ratio based on the similarity of the energy barriers would be $\sim 1$, which differs from the experimental data. This difference 
can be explained assuming that photodissociation takes place on the $S_{o}$ surface (after $S_{1} \rightarrow S_{o}$ internal conversion [29]) via a transition state geometrically connected with the trans conformer; and the transition state is formed before the vibrationally excited molecule statistically redistributes its vibrational energy [29]. Recently, we have shown that the photolysis of formic acid can be considered a clear case of isomer-selective reactions [33]. cis-Formic acid was prepared in an Ar matrix by excitation of the $2 \mathrm{vOH}$ mode of trans-formic acid and the branching ratio was determined via simultaneous IR pumping $\left(\approx 6930 \mathrm{~cm}^{-1}\right)$ and UV photolysis (193 nm) (see Fig. 6). In this situation, both conformers are present in the matrix during the UV irradiation and the production of $\mathrm{CO}_{2}$ increases by a factor of 4 when compared to the $193 \mathrm{~nm}$ photolysis of trans-formic acid alone. A simple spectral subtraction procedure allowed to extract the $\mathrm{CO} / \mathrm{CO}_{2}$ ratio for the photolysis of $c i s$-formic acid [33] (see Fig. 6). The proportion of the $\mathrm{CO}_{2}$ product is as high as $72 \%$ for the UV photolysis of the cis conformer, which corresponds to a branching ratio of $\approx 0.4$. This represents a change by more than one order of magnitude between the photolysis branching ratios of the two conformers.

The difference in the photodissociation process of the formic acid conformers is further supported by the band shapes of the $\mathrm{CO}_{2}$ products. While the isolated $\mathrm{CO}_{2}$ resulting from the photolysis of trans-formic acid exhibits two sharp bands, a broad $\mathrm{CO}_{2}$ band is obtained from the cis form. The product of trans-formic acid photolysis seems to be essentially monomeric $\mathrm{CO}_{2}$ isolated in a double-substitutional site [33]. On the other hand, the broadening of the $\mathrm{CO}_{2}$ band obtained from the cis conformer might result from $\mathrm{CO}_{2}$ complexed with $\mathrm{H}_{2}$ [33]. This difference can be understood by assuming that the $\mathrm{H}_{2}$ molecules produced from the cis photolysis do not have enough kinetic energy to leave the matrix cage. On the other hand, kinetically hot $\mathrm{H}_{2}$ molecules are suggested to be produced in the photolysis of the trans

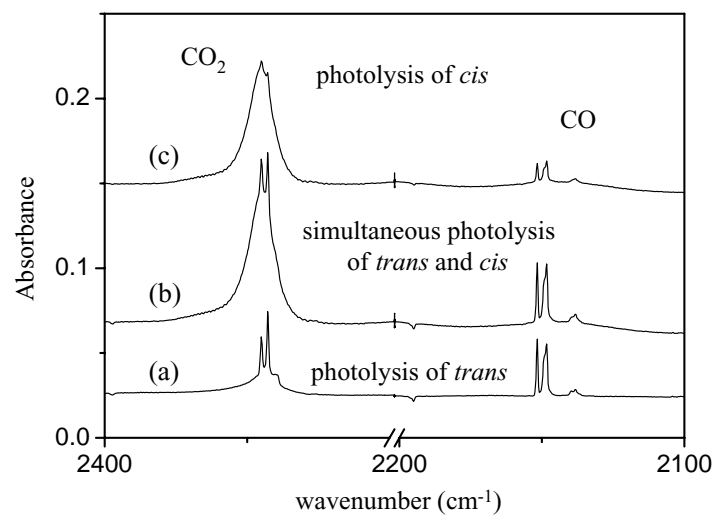

Fig. 6. Photolysis products $\left(\mathrm{CO}_{2}\right.$ and $\left.\mathrm{CO}\right)$ of formic acid isolated in $\mathrm{Ar}$ at $8 \mathrm{~K}$ : (a) resulting from the $193 \mathrm{~nm}$ irradiation of trans-formic acid; (b) resulting from the simultaneous $193 \mathrm{~nm}$ and $6930 \mathrm{~cm}^{-1}$ irradiation of formic acid isolated in $\mathrm{Ar}$ (the trans $\rightarrow$ cis isomerization is induced under IR irradiation) and (c) resulting from the $193 \mathrm{~nm}$ irradiation of the cis conformer, obtained by subtraction of (a) from (b). conformer leading to the cage exit of $\mathrm{H}_{2}$, thus preventing its complexation with $\mathrm{CO}_{2}$.

\section{Photoinduced rotamerization in dicarboxylic acids}

Excitation of the $2 \mathrm{vOH}$ vibration or a combination mode at similar energies was used to study rotamerization processes in dicarboxylic acids [34-36]. The four dicarboxylic acids studied are: ethanedioic, propanedioic, and $(E)$ - and (Z)-2-butenedioic acid (see Fig. 7). In dicarboxylic acids, an intramolecular hydrogen bond involving the hydroxyl moiety of one of the carboxylic groups and the carbonyl oxygen of the other group can be formed. This intramolecular hydrogen bond leads to stabilization of the $180^{\circ} \mathrm{O}=\mathrm{C}-$ $\mathrm{O}-\mathrm{H}$ dihedral angle. In general, the loss of electron density in the $\mathrm{O}-\mathrm{H}$ bond due to the intramolecular hydrogen bonding leads to a decrease in the corresponding force constant, and consequently to a redshift of the associated $\mathrm{O}-\mathrm{H}$ stretching mode.

Ethanedioic acid (also known as oxalic acid) is the smallest of the studied dicarboxylic acids. According to quantum chemical calculations [41-43] oxalic acid can have at least five conformers. The two lowest energy forms were earlier identified and isomerization between them was successfully promoted by both broadband-IR and UV irradiation [41], although these processes were not very efficient. In our studies, the three lowest energy conformers of oxalic acid were detected in the as-deposited Ar matrix with estimated energy differences $\left(\Delta E_{\mathrm{I}}: \Delta E_{\mathrm{II}}: \Delta E_{\mathrm{III}}\right)$ of $\left(0: 836: 1338 \mathrm{~cm}^{-1}\right)$ [34], see Fig. 7 . The photoinduced rotamerization experiments allowed a detailed characterization of the mid-IR spectra of the previously observed forms I and II [34], as well as that of form III, which was detected for the first time in our work [34].

The relative energies of the three observed oxalic acid conformers can be understood on the basis of intramolecular hydrogen bonding and electrostatic repulsion between the negative charge densities localized at the oxygen atoms. Conformers I and II, bearing the relatively strong $\mathrm{C}=\mathrm{O} \ldots \mathrm{H}-\mathrm{O}$ hydrogen bonds are more stable than conformer III. On the other hand, both the stronger type of intramolecular hydrogen bonds $[\mathrm{C}=\mathrm{O} \ldots \mathrm{H}-\mathrm{O}$ stronger than $\mathrm{C}-\mathrm{O}(\mathrm{H}) \ldots \mathrm{H}-\mathrm{O}]$ and the less important electrostatic effects between the lone electron pairs of the oxygen atoms $(=\mathrm{O} \ldots \mathrm{OH}$ weaker repulsive interaction comparing to $\mathrm{HO} \ldots \mathrm{OH}$ and $=\mathrm{O} \ldots \mathrm{O}=$ ) lead to a lower energy for the trans arrangement around the $\mathrm{C}-\mathrm{C}$ bond. This is the arrangement found in all of the three lowest energy conformers of oxalic acid.

Irradiation at $6712 \mathrm{~cm}^{-1}$ converts the most stable conformer into forms II and III. A further irradiation at $6755 \mathrm{~cm}^{-1}$ induces the II $\rightarrow$ I and II $\rightarrow$ III processes [34]. Identification of the conformers based on their IR absorption spectra was straightforward due to the high efficiency of the photoinduced processes [34]. This is illustrated in Fig. 8, 
(a) oxalic acid

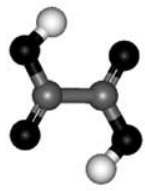

Form I

$0.0 \mathrm{~cm}^{-1}$

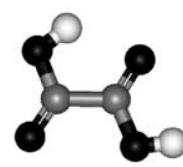

Form II

$836 \mathrm{~cm}^{-1}$

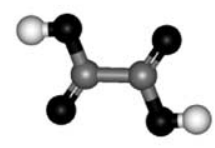

Form III

$1338 \mathrm{~cm}^{-1}$

(b) malonic acid

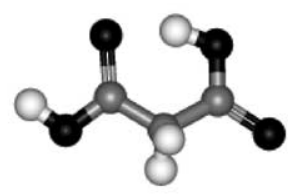

Form I $0.0 \mathrm{~cm}^{-1}$

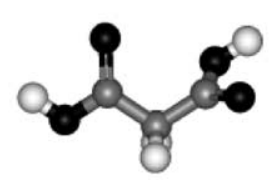

Form II $23 \mathrm{~cm}^{-1}$

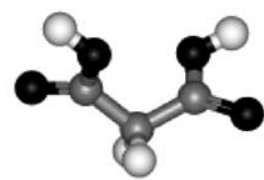

Form III

(c) maleic acid

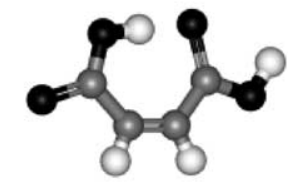

Form I $0 \mathrm{~cm}^{-1}$

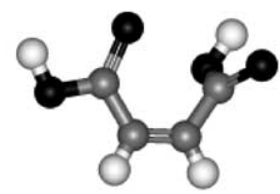

Form II

(d) fumaric acid

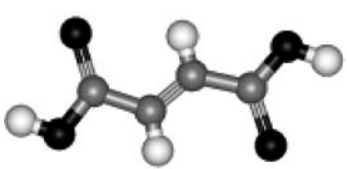

Form I

$0 \mathrm{~cm}^{-1}$

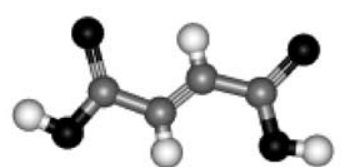

Form II

$50 \mathrm{~cm}^{-1}$

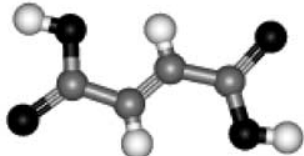

Form III

$117 \mathrm{~cm}^{-1}$

Fig. 7. Dicarboxylic acid conformers detected in the argon matrices and the corresponding estimated energy differences (in $\mathrm{cm}^{-1}$ ). For all but malonic acid the energy differences presented were calculated on the basis of the observed relative population in the as-deposited matrices [33,35]. For malonic acid the computationally predicted value is given [34].

where the $\mathrm{OH}$ and $\mathrm{C}=\mathrm{O}$ stretching bands in the relevant difference spectra are presented. The lower trace shows the spectrum of the as-deposited oxalic acid in an Ar matrix, where only the bands of the conformational ground state (form I) are clearly visible. The middle difference spectrum shows the result of excitation of form I at $6712 \mathrm{~cm}^{-1}$. The top trace of Fig. 8 presents the net result of irradiation of form I for about $1 \mathrm{~h}$ followed by pumping periodically forms I and II (at $6755 \mathrm{~cm}^{-1}$ ) during ca. $30 \mathrm{~min}$. In this case, the difference spectrum shows the growth of the absorption bands of conformer III while those belonging to conformers I and II decrease.

No evidence was found of photoinduced rotamerization involving rotation around the $\mathrm{C}-\mathrm{C}$ bond, despite its theoretically predicted lower barrier for conformer II than that estimated for the observed II $\rightarrow$ III rotamerization [34]. This result can be understood if the energy barrier to internal rotation around the $\mathrm{C}-\mathrm{C}$ bond for the free molecule differs strongly from that for the molecule isolated in the matrices. Indeed, rotation around the $\mathrm{C}-\mathrm{C}$ bond involves extensive motion of heavy atoms and this should require reorganization of the matrix atoms around the guest molecule. This can lead to a considerable increase of the energy required for the isomerization. Another possibility is an inefficient relaxation mechanism to transfer the energy deposited into the $2 \mathrm{vOH}$ mode to the $\mathrm{C}-\mathrm{C}$ torsion. However, the latter hypothesis is not supported either by the results obtained for formic acid, already described in this paper, or the results obtained for two of the studied carboxylic acids (where $\mathrm{C}-\mathrm{C}$ rotation was observed after $2 \mathrm{vOH}$ excitation).

The propanedioic acid (malonic acid) molecule was detected in three different forms, shown in Fig. 7, in an as-deposited Ar matrix [35]. Near-IR irradiation of the matrix produced a significant rearrangement of the conformational distribution, which enabled the assignment of the bands to the three lowest energy conformers predicted 


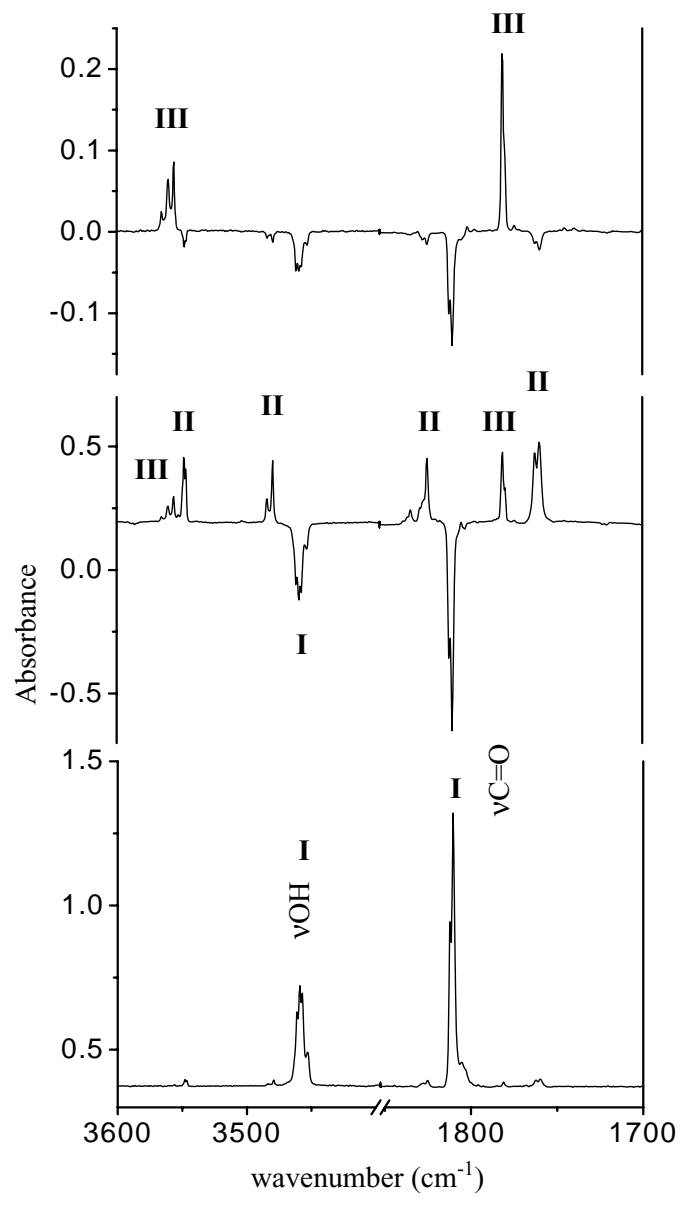

Fig. 8. IR absorption spectra $\left(3600-3400 \mathrm{~cm}^{-1}\right.$ and $1850-1700 \mathrm{~cm}^{-1}$ ) showing the result of near-IR irradiation of oxalic acid isolated in solid argon at $7.5 \mathrm{~K}$. The lower plot shows the spectrum of the as-deposited sample where the main bands correspond to the $\mathrm{OH}$ and $\mathrm{C}=\mathrm{O}$ stretching absorptions of the conformational ground state (form I). The middle plot is a difference spectrum where the bands belonging to form I point downwards and the bands of forms II and III are pointing upwards. The upper plot is a difference spectrum showing the bands of forms I and II pointing downwards and the bands of form III pointing upwards. See text for the pumping details.

theoretically. In analogy with oxalic acid, the most stable conformer of malonic acid is stabilized by intramolecular hydrogen bonding. The redshift induced by the intramolecular hydrogen bond of malonic acid is about four times larger $\left(295 \mathrm{~cm}^{-1}\right)$ than that of oxalic acid $\left(70 \mathrm{~cm}^{-1}\right)$.

The observed interconversion processes involved rotation around the $\mathrm{C}-\mathrm{O}$ bond as well as around the $\mathrm{C}-\mathrm{C}$ bond. This means that the restrictions imposed by the matrix to the rotation around the $\mathrm{C}-\mathrm{C}$ bond are less severe in malonic acid than in oxalic acid. This difference could be associated with the fact that malonic acid is a bigger molecule with all relevant conformers being non-planar. Hence a less compact arrangement of the lattice atoms around the malonic acid molecules can be expected to allow the heavy atoms to move, contrary to the case of the small planar oxalic acid.
The IR absorption spectrum of (Z)-2-butenedioic acid (fumaric acid) isolated in Ar was also interpreted in terms of the three lowest energy conformers predicted theoretically [36] and shown in Fig. 7. As expected on the basis of the previous studies on $\alpha, \beta$-unsaturated molecules [44], the relative conformational energy in fumaric acid increases with the number of trans arrangements around the $\mathrm{C}_{\alpha}-\mathrm{C}$ bonds. On the other hand, in the case of maleic acid [(E)-2-butenedioic acid] the cis arrangement around both $\mathrm{C}_{\alpha}-\mathrm{C}$ bonds is not favored due to the strong repulsive interaction between the lone pairs of the carbonylic oxygen atoms (see Fig. 7). Hence, for this molecule the most stable conformer has one cis and one trans conformation along the $\mathrm{C}_{\alpha}-\mathrm{C}$ axis and it is stabilized by a very strong $\mathrm{OH} \ldots \mathrm{O}=$ intramolecular hydrogen bond (the observed redshift of the $\mathrm{vOH}$ vibration frequency associated with the intramolecularly bonded hydroxyl group is as large as $440 \mathrm{~cm}^{-1}$ [36]). The second lowest energy conformer of maleic acid possesses a non-planar geometry with two nearly perpendicular carboxylic groups, which minimizes the electrostatic and steric repulsions between these groups.

Interconversion between the two lowest energy conformers of maleic acid was induced by IR excitation and also by annealing the matrix at $35 \mathrm{~K}$ [36]. As expected, annealing of the matrix resulted in conversion from the less stable form II to the conformational ground state (form I) [36]. Tuning the IR radiation over the $6940-6835 \mathrm{~cm}^{-1}$ region allowed us to find only one mode, at $6901 \mathrm{~cm}^{-1}$, which was active to promote rotamerization. This mode corresponds to the $2 \mathrm{vOH}$ vibration of form II and it promotes the II $\rightarrow$ I rotamerization. This isomerization process in maleic acid involves rotation around both the $\mathrm{C}-\mathrm{O}$ and $\mathrm{C}_{\alpha}-\mathrm{C}$ bonds. It should be stressed that we were able to induce rotamerization starting from the non-planar conformer (form II) but we did not succeed to induce any isomerization processes from the planar conformer (form I). If the anharmonicity of the $2 \mathrm{vOH}$ mode in form I is similar to that of the same vibration in conformer II, then form I absorbs within the probed spectral region. Hence, this observation further supports the results previously discussed for both oxalic and malonic acids that, for the planar conformers, the energy barrier to rotation around the $\mathrm{C}-\mathrm{C}$ bond seems to increase considerably in matrices as compared with the gas phase. This observation is also consistent with the idea that the reaction barrier for conformational isomerization processes may be considerably different depending on the nature of the cage occupied by the trapped molecule. A more compact trapping site for the planar conformers would imply a higher energy barrier for the rotamerization while the non-planar conformers isolated in bigger cages would have a lower rotamerization barrier.

In the case of fumaric acid, no rotamerization was found while IR irradiation was scanned through the 7018$6873 \mathrm{~cm}^{-1}$ spectral region. From the IR-pumping experiments performed on the other dicarboxylic acids studied, we 
were expecting to find at least two active $2 \mathrm{vOH}$ modes in this region belonging to form II (in forms I and III this mode is infrared inactive due to symmetry restrictions). The annealing experiments were equally inefficient in inducing rotational isomerization processes in fumaric acid. Since conformational interconversion between the three observed conformers of fumaric acid should occur through internal rotation around the $\mathrm{C}_{\alpha}-\mathrm{C}$ bonds, as for the previous molecules, this negative observation is also consistent with an increased barrier to rotation around the $\mathrm{C}_{\alpha}-\mathrm{C}$ bond for the planar conformers in matrices. On the other hand, internal rotation around the $\mathrm{C}-\mathrm{O}$ bonds of fumaric acid would lead to high energy conformers, with energies higher than the ground conformational state by $2100 \mathrm{~cm}^{-1}$. This situation contrasts with that for the other molecules studied where conformers differing by internal rotation around the $\mathrm{C}-\mathrm{O}$ bonds are relatively close in energy. Though these high energy species could eventually be formed, they could convert back by tunneling to the more stable forms fast enough to prevent their detection under our experimental conditions $[19,36]$.

\section{Concluding remarks}

In this work, an overview of our recent studies dealing with infrared-induced conformational interconversion of carboxylic acids isolated in rare-gas matrices was presented. Photoreactive spectroscopy (narrowband IR pumping coupled with vibrational spectroscopy) is a powerful method to investigate conformational properties of matrix-isolated molecules. This method allowed us to probe the local morphology effects on several intermode couplings and anharmonicity constants by investigating the site-resolved vibrational spectroscopy of formic acid isolated in solid Ar.

The trans $\rightarrow$ cis isomerization reaction induced by modeselective irradiation of trans-formic acid was studied. The excitation energy was quite efficiently relaxed into the reaction coordinate $(\mathrm{C}-\mathrm{O}$ torsion) from all vibrationally excited levels used here. Tunneling was found to play an important role in the trans $\rightarrow$ cis photoinduced reaction mechanism. On the other hand, phonon-assisted tunneling mechanism was demonstrated on the reverse cis $\rightarrow$ trans process as well. In this case, a strong dependence of the reaction rate on both temperature and solid host was found. The local morphology within the same host was also shown to influence the tunneling rate. From these studies it is possible to conclude that the tunneling mechanism can contribute in similar isomerization reactions for various molecules bearing a hydroxyl group. Photodissociation of formic acid was shown to be an isomer-selective reaction, where the time scale for rotamerization is longer than for dissociation. Narrowband IR irradiation was shown to be an effective switch between the two main reaction channels of formic acid UV-photolysis (decarboxylation and dehydration) by changing the cis/trans ratio in the irradiated matrix.
Using near-IR irradiation we were able to promote conformational interconversion in a series of dicarboxylic acids. Excitation of the first $\mathrm{O}-\mathrm{H}$ stretching overtone leads to internal rotation around the $\mathrm{C}-\mathrm{O}$ bond in oxalic, malonic and maleic acid but does not isomerize fumaric acid. Rotamerization around the $\mathrm{C}-\mathrm{C}$ bond was observed only when the precursors for IR-pumping were non-planar, possibly due to the effect of a more restrictive packing of the lattice atoms around the planar conformers that hinders the internal rotation when it involves an extensive motion of heavy atoms.

\section{Acknowledgements}

The Academy of Finland and the Portuguese Foundation for Science and Technology are thanked for financial support.

\section{References}

[1] A.J. Barnes, J. Mol. Struct. 113 (1984) 161.

[2] M. Räsänen, H. Kunttu, J. Murto, Laser Chem. 9 (1988) 123.

[3] T. Lotta, J. Murto, M. Räsänen, A. Aspiala, J. Mol. Struct. 114 (1984) 333.

[4] H.H. Günthard, J. Mol. Struct. 113 (1984) 141.

[5] R.N. Perutz, Chem. Rev. 85 (1985) 97.

[6] H. Frei, G.C. Pimentel, Chemistry and Physics of Matrix-Isolated Species, in: L. Andrews, M. Moskovits (Eds.), Elsevier B.V., Amsterdam, 1989, p. 139.

[7] M. Poliakoff, J.J. Turner, Chemical and Biochemical Applications of Lasers, in: C.B. Moore (Ed.), vol. 5, 1980, p. 175.

[8] H. Frei, G.C. Pimentel, Annu. Rev. Phys. Chem. 36 (1985) 491.

[9] S. Kudoh, M. Takayanagi, M. Nakata, T. Ishibashi, M. Tasumi, J. Mol. Struct. 479 (1999) 41.

[10] A.K. Knudsen, G.C. Pimentel, J. Phys. Chem. 95 (1991) 2823.

[11] J. Pourcin, G. Davidovics, H. Bodot, L. Abouaf-Marguin, B. Gauthier-Roy, Chem. Phys. Lett. 74 (1980) 147.

[12] W.F. Hoffman III, J.S. Shirk, Chem. Phys. 78 (1983) 331.

[13] P. Roubin, S. Varin, P. Verlaque, S. Coussan, J.-M. Berset, J.-M. Ortéga, A. Peremans, W.-Q. Zheng, J. Chem. Phys. 107 (1997) 7800 .

[14] S. Coussan, Y. Bouteiller, J.P. Perchard, W.Q. Zheng, J. Phys. Chem. A 102 (1998) 5789.

[15] S. Sander, H. Willner, L. Khriachtchev, M. Pettersson, M. Räsänen, E.L. Varetti, J. Mol. Spectrosc. 203 (2000) 145.

[16] L. Khriachtchev, J. Lundell, E. Isoniemi, M. Räsänen, J. Chem. Phys. 113 (2000) 4265.

[17] K.B. Wiberg, K.E. Laidig, J. Am. Chem. Soc. 109 (1987) 5935.

[18] M. Pettersson, J. Lundell, L. Khriachtchev, M. Räsänen, J. Am. Chem. Soc. 119 (1997) 11715.

[19] M. Pettersson, E.M.S. Maçôas, L. Khriachtchev, J. Lundell, R. Fausto, M. Räsänen, J. Chem. Phys. 117 (2002) 9095.

[20] M. Pettersson, E.M.S. Maçôas, L. Khriachtchev, R. Fausto, M. Räsänen, J. Am. Chem. Soc. 125 (2003) 4058.

[21] N. Akai, S. Kudoh, M. Takayanagi, M. Nakata, Chem. Phys. Lett. 356 (2002) 133.

[22] N. Akai, S. Kudoh, M. Takayanagi, M. Nakata, J. Phys. Chem. 106 (2002) 11029.

[23] V.A. Benderskii, D.E. Makarov, C.A. Wight, in: Chemical Dynamics at Low Temperature, Wiley, New York, 1994. 
[24] G.K. Ivanov, M.A. Kozhushner, L.I. Trakhtenberg, J. Chem. Phys. 113 (2000) 1992.

[25] M.V. Basilevsky, G.V. Davidovitch, J. Chem. Phys. 115 (2001) 6072.

[26] R. Silbey, H.-P. Trommsdorff, Chem. Phys. Lett. 165 (1990) 540.

[27] A. Suárez, R.J. Silbey, Chem. Phys. 94 (1991) 4809.

[28] T. Kumada, K. Komaguchi, Y. Aratono, T. Miyazaki, Chem. Phys. Lett. 261 (1996) 463.

[29] H. Su, Y. He, F. Kong, W. Fang, R. Liu, J. Chem. Phys. 113 (2000) 1891.

[30] S.T. Park, S.K. Kim, M.S. Kim, Nature 415 (2002) 306.

[31] S.T. Park, M.S. Kim, J. Chem. Phys. 117 (2002) 124.

[32] E.M.S. Maçôas, J. Lundell, M. Pettersson, L. Khriachtchev, R. Fausto, M. Räsänen, J. Mol. Spectrosc. 219 (2003) 70.

[33] L. Khriachtchev, E. Maçôas, M. Pettersson, M. Räsänen, J. Am. Chem. Soc. 124 (2002) 10994.

[34] E.M.S. Maçôas, R. Fausto, M. Pettersson, L. Khriachtchev, M. Räsänen, J. Phys. Chem. A 104 (2000) 6956.
[35] E.M.S. Maçôas, R. Fausto, J. Lundell, M. Pettersson, L. Khriachtchev, M. Räsänen, J. Phys. Chem. A 104 (2000) 11725.

[36] E.M.S. Maçôas, R. Fausto, J. Lundell, M. Pettersson, L. Khriachtchev, M. Räsänen, J. Phys. Chem. A 105 (2001) 3922.

[37] M. Freytes, D. Hurtmans, S. Kassi, J. Liévin, J. Vander Auwera, A. Campargue, M. Herman, Chem. Phys. 283 (2002) 47.

[38] V.E. Bondybey, Adv. Chem. Phys. 47 (1981) 521.

[39] J. Goddard, Y. Yamaguchi, H.F. Schaefer III, J. Chem. Phys. 96 (1992) 1158.

[40] J. Lundell, M. Räsänen, J. Mol. Struct. 436-437 (1997) 349.

[41] J. Nieminen, M. Räsänen, J. Murto, J. Phys. Chem. 96 (1992) 5303.

[42] J. Higgins, X. Zhou, R. Liu, T.T.-S. Huang, J. Phys. Chem. A 101 (1997) 2702.

[43] P.D. Godfrey, M.J. Mirabella, R.D. Brown, J. Phys. Chem. A 104 (2000) 258.

[44] A. Kulbida, M.N. Ramos, M. Räsänen, J. Nieminen, O. Schrems, R. Fausto, J. Chem. Soc. Faraday Trans. 91 (1995) 1571. 